\title{
THE SECOND CENTRAL MOMENT OF ADDITIVE FUNCTIONS
}

\author{
JUNGSEOB LEE
}

(Communicated by William Adams)

Abstract. We prove that the best constant in the Turán-Kubilius inequality for additive functions is $3 / 2$ in any sufficiently large range.

\section{THE RESULT}

A complex-valued function $f$ defined on the positive integers is called additive if $f(m n)=f(m)+f(n)$ for any coprimes $m$ and $n$. For any additive function $f$ and any real number $x \geq 2$, we define

$$
A(f, x)=\sum_{p^{a} \leq x} \frac{f\left(p^{a}\right)}{p^{a}}\left(1-\frac{1}{p}\right)
$$

and

$$
D(f, x)=\left(\sum_{p^{a} \leq x} \frac{\left|f\left(p^{a}\right)\right|^{2}}{p^{a}}\right)^{1 / 2} .
$$

Here and throughout $p^{a}$ denote powers of primes $p$. We also define

$$
\tau(f, x)=\frac{1}{x D(f, x)^{2}} \sum_{n \leq x}|f(n)-A(f, x)|^{2}
$$

and

$$
\tau(x)=\sup \tau(f, x),
$$

where the supremum is taken over all the additive functions such that $D(f, x)>$ 0 . The celebrated Turán-Kubilius inequality asserts that $\tau(x)$ is bounded, and to find the best bound has been a persistent problem for a long period of time. Since Kubilius [5, 6] proved that $\tau(x)=3 / 2+O\left(\log ^{-1 / 2} x\right)$ (see Hildebrand [3] and Stein [8] for independent proofs of similar results and Elliott [2] for a comprehensive discussion on the subject), it has been questioned if $3 / 2$ really is the best constant in the Turán-Kubilius inequality. Subsequently Lee [7] proved that this is the case for strongly additive functions and all sufficiently large $x$ by elaborating on Kubilius's argument. In this paper we generalize this result to additive functions. More precisely, we prove the following result.

Received by the editors August 23, 1990.

1980 Mathematics Subject Classification (1985 Revision). Primary 11K65. 
Theorem. There exists absolute positive constants $c$ and $d$ such that

$$
\frac{3}{2}-\frac{c}{\log x} \leq \tau(x) \leq \frac{3}{2}-\frac{d}{\log x}
$$

for all sufficiently large $x$. Moreover this holds for any $d \leq 0.644 \ldots$.

Remarks. The constants $c$ and $d$ in the theorem can be calculated explicitly, but we will not pursue their values in this note. It is sometimes useful in number theory to assert the Turán-Kubilius inequality with $A(f, x)$ replaced by $A_{1}(f, x)=\sum_{p^{a} \leq x} f\left(p^{a}\right) / p^{a}$. We should note that the whole argument in this paper holds with a slight modification and the theorem is still true for $\tau(x)$ with $A_{1}(f, x)$ in place of $A(f, x)$.

As usual, we let $[u]$ denote the largest integer not exceeding the real number $u$ and let $\{u\}$ be the fractional part of $u,\{u\}=u-[u]$. For any real number $u \neq 0$, the fractional part of $x / u$ is denoted by $\rho_{x}(u)$ or simply by $\rho(u)$. Generally $p^{a}$ and $q^{b}$ denote powers of primes. We write $p^{a} \| n$ when $p^{a}$ divides $n$ but $p^{a+1}$ does not.

For the proof of the theorem, first we need to obtain a new representation for $\tau(f, x)$ defined in (1.1). Throughout the proof we will assume that $x$ is an integer, since we may do so. Observe that the additivity of $f$ yields

$$
\begin{aligned}
\sum_{n \leq x} f(n) & =\sum_{p^{a} \leq x} f\left(p^{a}\right) \sum_{\substack{n \leq x \\
p^{a} \| n}} 1 \\
& =\sum_{p^{a} \leq x} f\left(p^{a}\right)\left(\left[\frac{x}{p^{a}}\right]-\left[\frac{x}{p^{a+1}}\right]\right) .
\end{aligned}
$$

We also observe that

$$
\begin{aligned}
\sum_{n \leq x}|f(n)|^{2}=\sum_{p^{a} \leq x}\left|f\left(p^{a}\right)\right|^{2} \sum_{\substack{n \leq x \\
p^{a} \| n}} 1+\sum_{\substack{p^{a} q^{b} \leq x \\
p \neq q}} f\left(p^{a}\right) \bar{f}\left(q^{b}\right) \sum_{\substack{n \leq x \\
p^{a}\left\|n, q^{b}\right\| n}} 1 \\
=\sum_{p^{a} \leq x}\left|f\left(p^{a}\right)\right|^{2}\left(\left[\frac{x}{p^{a}}\right]-\left[\frac{x}{p^{a+1}}\right]\right) \\
\quad-\sum_{p^{a+b} \leq x} f\left(p^{a}\right) \bar{f}\left(p^{b}\right)\left(\left[\frac{x}{p^{a+b}}\right]-2\left[\frac{x}{p^{a+b+1}}\right]+\left[\frac{x}{p^{a+b+2}}\right]\right) \\
\quad+\sum_{p^{a} q^{b} \leq x} f\left(p^{a}\right) \bar{f}\left(q^{b}\right)\left(\left[\frac{x}{p^{a} q^{b}}\right]-\left[\frac{x}{p^{a+1} q^{b}}\right]-\left[\frac{x}{p^{a} q^{b+1}}\right]+\left[\frac{x}{p^{a+1} q^{b+1}}\right]\right) .
\end{aligned}
$$

We put

$$
x\left(p^{a}\right)=D(f, x)^{-1} p^{-a / 2} f\left(p^{a}\right)
$$

so that

$$
\sum_{p^{a} \leq x}\left|x\left(p^{a}\right)\right|^{2}=1 .
$$

By squaring out the right-hand side of (1.1) and using (1.2), (1.3) along with the normalization (1.4), we obtain

$$
\tau(f, x)=P-Q,
$$


where

$$
\begin{aligned}
& P=\frac{1}{x} \sum_{p^{a} \leq x}\left(\left[\frac{x}{p^{a}}\right]-\left[\frac{x}{p^{a-1}}\right]\right) p^{a}\left|x\left(p^{a}\right)\right|^{2}-\sum_{p^{a+b} \leq x} \frac{x\left(p^{a}\right)}{p^{a / 2}} \frac{\bar{x}\left(p^{b}\right)}{p^{b / 2}}\left(1-\frac{1}{p}\right)^{2} \\
& +\frac{1}{x} \sum_{p^{a+b} \leq x}\left(\rho\left(p^{a+b}\right)-2 \rho\left(p^{a+b+1}\right)+\rho\left(p^{a+b+2}\right)\right) p^{a / 2} p^{b / 2} x\left(p^{a}\right) \bar{x}\left(p^{b}\right) ; \\
& Q=\sum_{p^{a}, q^{b} \leq x} c\left(p^{a}, q^{b}\right) x\left(p^{a}\right) \bar{x}\left(q^{b}\right) \\
& c=c_{1}+c_{2}-c_{3}-c_{4}-c_{5}+c_{6}-c_{7}-c_{8}-c_{9} \\
& c_{1}\left(p^{a}, q^{b}\right)=\left\{\begin{array}{l}
p^{-a / 2} q^{-b / 2} \quad \text { if } p^{a} q^{b}>x \\
0 \text { otherwise }
\end{array}\right. \\
& c_{2}\left(p^{a}, q^{b}\right)=\left\{\begin{array}{l}
\frac{1}{x} \rho\left(p^{a} q^{b}\right) p^{a / 2} q^{b / 2} \text { if } p^{a} q^{b} \leq x \\
0 \text { otherwise }
\end{array}\right. \\
& c_{3}\left(p^{q}, q^{b}\right)=\frac{1}{x}\left(\rho\left(p^{a}\right) p^{a / 2} q^{-b / 2}+\rho\left(q^{b}\right) p^{-a / 2} q^{b / 2}\right) \\
& c_{4}\left(p^{a}, q^{b}\right)= \begin{cases}p^{-a / 2-1} q^{-b / 2}+q^{-a / 2} q^{-b / 2-1} & \text { if } p^{a} q^{b}>x, \\
0 & \text { otherwise }\end{cases} \\
& c_{5}\left(p^{a}, q^{b}\right)= \begin{cases}\frac{1}{x}\left(\rho\left(p^{a+1} q^{b}\right)+\rho\left(p^{a} q^{b+1}\right)\right) p^{a / 2} q^{b / 2} & \text { if } p^{a} q^{b} \leq x, \\
0 & \text { otherwise }\end{cases} \\
& c_{6}\left(p^{a}, q^{b}\right)=\frac{1}{x}\left(\rho\left(p^{a}\right) p^{a / 2} q^{-b / 2-1}+\rho\left(q^{b}\right) p^{-a / 2-1} q^{b / 2}\right) \\
& c_{7}\left(p^{a}, q^{b}\right)=\left\{\begin{array}{l}
p^{-a / 2-1} q^{-b / 2-1} \\
0 \text { otherwise }
\end{array} \text { if } p^{a} q^{b}>x,\right. \\
& c_{8}\left(p^{a}, q^{b}\right)= \begin{cases}\frac{1}{x} \rho\left(p^{a+1} q^{b+1}\right) p^{a / 2} q^{b / 2} & \text { if } p^{a} q^{b} \leq x, \\
0 \text { otherwise } & \end{cases} \\
& c_{9}\left(p^{a}, q^{b}\right)=\frac{1}{x}\left(\rho\left(p^{a+1}\right) p^{a / 2} q^{-b / 2}\left(1-\frac{1}{q}\right)+\rho\left(q^{b+1}\right) p^{-a / 2} q^{b / 2}\left(1-\frac{1}{p}\right)\right) .
\end{aligned}
$$

It can be shown by elementary arguments that $P \leq 1+O\left(x^{-1 / 4} \log x\right)$. For details see Kubilius [6]. In order to prove the theorem, it remains to find an appropriate lower bound for $Q$, which is the main body of the proof. In the next section we will prove the preliminary arithmetic lemmas, and in $\S 3$ we will complete the proof of the theorem.

\section{THE LEMMAS}

A form of the prime number theorem states that

$$
\sum_{p^{a} \leq x} 1=\int_{2}^{x} \frac{d x}{\log x}+R(x),
$$

where the error term is $R(x) \ll x \exp (-c \sqrt{\log x}), c>0$. In this section the constant $c$ may not be the same in each occurrence. Using summation by parts and (2.1), we can easily prove the following lemma. 
Lemma 1. For any positive integer $k$ and any $x \geq 2$,

$$
\sum_{p^{a} \leq x} \frac{\log ^{k} p^{a}}{p^{a}}=\frac{1}{k} \log ^{k} x+\delta_{k}+O_{k}(\exp (-c \sqrt{\log x})),
$$

where $\delta_{k}$ is a constant that depends only on $k$.

Lemma 2. For any real $x \geq 5$,

(i) $\sum_{p^{a}, q^{b} \leq x} \log q^{b} / p^{a} q^{b}=\log x+O(1)$,

(ii) $\sum_{p^{a}, q^{b} \leq x}^{p^{a}{ }^{b}>x} \log ^{2} q^{b} / p^{a} q^{b}=\frac{3}{4} \log ^{2} x+O(\log x)$. $p^{a} q^{b}>x$

Proof. We will prove the first assertion, since the second one can be proved analogously. Treating the summation over $q^{b}$ by Lemma 1 , the sum in (i) becomes

$$
\sum_{p^{a} \leq x} \frac{\log p^{a}}{p^{a}}+O\left(\sum_{p^{a} \leq x} \frac{1}{p^{a}} \exp \left(-c \sqrt{\log \left(x / p^{a}\right)}\right)\right) .
$$

The main term above is $\log x+O(1)$ by Lemma 1 , and the error term is « $1 / \log x$.

De la Vallée-Poussin [1] proved by an elementary method that

$$
\sum_{p \leq x} \rho(p) \sim \alpha \frac{x}{\log x},
$$

where $\alpha=1-c_{0}$ and $c_{0}$ is Euler's constant. Using complex integration, however, one can obtain expansions in negative powers of $\log x$ for the above sum and other similar sums.

Lemma 3. For any $x \geq 3$,

(i) $\sum_{p^{a} \leq x} \rho\left(p^{a}\right)=\alpha \frac{x}{\log x}+O\left(x / \log ^{2} x\right)$;

(ii) $\sum_{p^{a} \leq x} \rho\left(p^{a}\right) \log p^{a}=\alpha x+O(x \exp (-c \sqrt{\log x}))$;

(iii) $\sum_{p^{a} \leq x} \rho\left(p^{a}\right) \log ^{2} p^{a}=\alpha x \log x+O(x)$;

(iv) $\sum_{p^{a} \leq x} \rho\left(p^{a}\right)^{2} p^{a}=\beta \frac{x^{2}}{\log x}+O\left(x^{2} / \log ^{2} x\right)$,

where the constant $\beta=\frac{3}{2}-c_{0}-\frac{\pi^{2}}{12}$.

Proof. The sum in (i) is equal to

$$
\sum_{n \leq x} \rho(n) \frac{\Lambda(n)}{\log n}+O(\log x),
$$

and by Perron's formula, the last sum is

$$
\frac{1}{2 \pi i} \int(x \log \zeta(s+1)-\zeta(s) \log \zeta(s)) \frac{x^{s}}{s} d s,
$$

where the integral is over an appropriate vertical contour. Evaluating this integral by standard methods we get the desired result. For the details see Lee [7, p. 17]. 
The remaining sums can be estimated similarly. We note that the associated Dirichlet series for the sums in (ii), (iii), and (iv) are, respectively,

$$
\begin{gathered}
-x \frac{\zeta^{\prime}}{\zeta}(s+1)-\left(-\frac{\zeta^{\prime}}{\zeta}(s)\right) \zeta(s), \\
-x\left(-\frac{\zeta^{\prime}}{\zeta}(s+1)\right)^{\prime}+\left(-\frac{\zeta^{\prime}}{\zeta}(s)\right)^{\prime} \zeta(s), \\
x^{2} \log \zeta(s+1)-2 x \zeta(s) \log \zeta(s)+\zeta(s) \zeta(s-1) \log \zeta(s-1) .
\end{gathered}
$$

Remark. Although the proof of (i) by complex integration gives us the most accurate result, it involves the logarithmic singularities of the integrand. Avoiding this complication, we may derive (i) from (ii) using the inequality

$$
\sum_{p^{a} \leq x} \rho\left(p^{a}\right)\left(\log x-\log p^{a}\right) \leq \sum_{p^{a} \leq x}\left(\log x-\log p^{a}\right)=O(x / \log x) .
$$

The sum in (iv) can be estimated rather elementarily using summation by parts and the prime number theorem, which requires dealing with jump discontinuities of the summand function.

Lemma 4. For any real number $x \geq 2$ and any integer $k \geq 0$,

(i) $\sum_{p^{a} \leq x} \rho\left(p^{a+1}\right) \log ^{k} p^{a} \ll x^{1 / 2} \log ^{k-1} x$;

(ii) $\sum_{p^{a} \leq x} \rho\left(p^{a+1}\right) p^{a / 2} \ll x^{3 / 4} / \log x$;

(iii) $\sum_{p^{a} \leq x} \rho\left(p^{a+1}\right) p^{a} \ll x^{3 / 2} / \log x$;

(iv) $\sum_{p^{2} \leq x} \rho\left(p^{a+1}\right)^{2} p^{a} \ll x^{4 / 3} / \log x$.

Proof. We will prove (i) since the others can be shown analogously. Distinguishing between $a=1$ and $a>1$, the sum to be estimated is

$$
S+T=\sum_{p \leq x} \rho\left(p^{2}\right) \log ^{k} p+\sum_{\substack{p^{a} \leq x \\ a>1}} \rho\left(p^{a+1}\right) \log ^{k} p^{a} .
$$

One can see that

$$
\begin{aligned}
T & \ll \sum_{p \leq \sqrt{x}} \log ^{k} p \sum_{1<a \leq \log x / \log p} a^{k} \\
& \ll \log ^{k+1} x \sum_{p \leq \sqrt{x}} \frac{1}{\log p} \ll \sqrt{x} \log ^{k-1} x .
\end{aligned}
$$

On the other hand, dividing the interval of the summation we write

$$
S=\sum_{p \leq \sqrt{x}} \rho\left(p^{2}\right) \log ^{k} p+x \sum_{\sqrt{x}<p \leq x} \frac{\log ^{k} p}{p^{2}} .
$$

It is clear that the first sum on the right is $\ll \sqrt{x} \log ^{k-1} x$ and the second one is $\ll\left(\log ^{k-1} x\right) / \sqrt{x}$. The proof is complete.

Lemma 5. For any integer $k \geq 0$ and any real $x>3$,

$$
\sum_{p^{a}>x} \frac{\log ^{k} p^{a}}{p^{a+1}} \ll \frac{\log ^{k} x \log \log x}{x} .
$$


Proof. Write the sum as

The first sum is

$$
\sum_{\substack{p^{a}>x \\ p \leq x}} \frac{\log ^{k} p^{a}}{p^{a+1}}+\sum_{\substack{p^{a}>x \\ p>x}} \frac{\log ^{k} p^{a}}{p^{a+1}} .
$$

$$
\sum_{p \leq x} \frac{\log ^{k} p}{p} \sum_{a>\log x / \log p} \frac{a^{k}}{p^{a}} \ll \frac{\log ^{k} x}{x} \sum_{p \leq x} \frac{1}{p} \ll \frac{\log ^{k} x \log \log x}{x},
$$

and the second one is

$$
\sum_{p>x} \frac{\log ^{k} p}{p} \sum_{a} \frac{a^{k}}{p^{a}} \ll \sum_{p>x} \frac{\log ^{k} p}{p^{2}} \ll \frac{\log ^{k-1} x}{x} .
$$

The proof is complete.

\section{THE PROOF}

It remains to find a lower bound for the bilinear form $Q$. Since the coefficient matrix $\mathbf{C}$ is real and symmetric, $Q$ is bounded from below by the smallest eigenvalue of $\mathbf{C}$. It was known to Kubilius [6] that the eigenvalues of $\mathbf{C}$ are

$$
\nu_{r}=(-1)^{r+1} / r+O\left(\log ^{-1 / 2} x\right), \quad r=1,2,3, \ldots
$$

with corresponding approximate eigenvectors $\Psi_{r}$ whose $p^{a}$ th component is $p^{-a / 2} \psi_{r}\left(\log p^{a} / \log x\right)$, where

$$
\psi_{r}(u)=u \sum_{k=0}^{r-1}(-1)^{r-1-k}\left(\begin{array}{c}
r \\
k
\end{array}\right)\left(\begin{array}{c}
r-1 \\
k
\end{array}\right) u^{r-k-1}(1-u)^{k} .
$$

By observing that $\sum\left|\nu_{r}\right|^{2}=\pi^{2} / 6+O\left(\log ^{-1 / 2} x\right)$, Kubilius also showed that when $x$ is sufficiently large, $\nu_{r}$, for small $r$, are all distinct and the smallest eigenvalue of the matrix $\mathbf{C}$ is $\nu_{2}$. We can exploit the estimates in $\S 2$, however, to show that the sum is $\pi^{2} / 6-\kappa \log \log x+O(1 / \log x)$ with positive constant $\kappa$, which suggests that the eigenvalues may be $(-1)^{r+1} / r+(-1)^{r} \kappa_{r} / \log x+$ $o(1 / \log x)$ with $\kappa_{r}>0$. In this paper our interest lies on $\nu_{2}$. We show that the smallest eigenvalue of $\mathrm{C}$ is greater than $-1 / 2+c \log x+O\left(\log \log ^{2} x / \log ^{2} x\right)$ with $c>0$ for all sufficiently large $x$. For this purpose we need the following fact. For the proof see Horn and Johnson [4, Theorem 6.3.14].

Lemma. Let $\mathbf{C}$ be a hermitian matrix, $\mathbf{x}$ be a vector, and $\mu$ a complex number. Then there exists an eigenvalue $\nu$ such that

$$
\|\mathbf{x}\||\nu-\mu| \leq\|\mathbf{C} \mathbf{x}-\mu \mathbf{x}\| .
$$

We will apply this lemma to the coefficient matrix $\mathbf{C}$ of the bilinear form $Q$ with $\mu=-1 / 2+A / \log x$, where $A$ is a number at our disposal and with $\mathbf{x}=\left(x\left(p^{a}\right)\right)$, where each component $x\left(p^{a}\right)$ is

$$
\begin{aligned}
x_{1}\left(p^{a}\right) & -x_{2}\left(p^{a}\right)+x_{3}\left(p^{a}\right)-x_{4}\left(p^{a}\right)-x_{5}\left(p^{a}\right)-x_{6}\left(p^{a}\right)+x_{7}\left(p^{a}\right) \\
= & \frac{4}{\log x} \frac{\log p^{a}}{p^{a / 2}}-\frac{6}{\log ^{2} x} \frac{\log ^{2} p^{a}}{p^{a / 2}}+\frac{2}{x} \rho\left(p^{a}\right) p^{a / 2}-\frac{4(\alpha-\beta)}{\log x} \frac{1}{p^{a / 2}} \\
& -\frac{4}{\log x} \frac{\log p^{a}}{p^{a / 2+1}}-\frac{4}{\log x} \frac{\log p}{p^{a / 2+1}}+\frac{4(\alpha-\beta)}{\log x} \frac{1}{p^{a / 2-1}} .
\end{aligned}
$$


It can be easily seen that

$$
\|\mathbf{x}\|^{2}=1+\frac{12 \beta-16 \alpha}{\log x}+O\left(\frac{\log \log x}{\log ^{2} x}\right)
$$

using Lemmas 1 and 3. We should note that this vector is a more precise approximate eigenvector corresponding to the lowest eigenvalue than Kubilius's $\Psi_{2}$, and can be calculated systematically using the techniques from numerical analysis; namely, the power method and deflation of matrix. In fact, the vector $x$ can be found from $\left(C-\Psi_{1} \Psi_{1}^{t}\right) \Psi_{2}$.

To evaluate the right-hand side of (3.1), we will calculate the $p^{a}$ th component of $\mathbf{C x}$. First note that by using Lemmas 4 and 5 it can be shown that $\left\|\mathbf{C}_{i} \mathbf{x}\right\| \ll$ $\log ^{-3 / 2} x$ for $i=7,8,9$. From here on $\mathbf{e}=\left(e\left(p^{a}\right)\right)$ denotes any vector with $\|\mathbf{e}\| \ll \log \log x / \log ^{3 / 2} x$.

Straightforward calculations using Lemmas 1, 3, 4, and 5 yield

$$
\begin{aligned}
\sum_{q^{b} \leq x} c\left(p^{a}, q^{b}\right) x_{1}\left(q^{b}\right)= & \frac{4}{\log x} \frac{\log p^{a}}{p^{a / 2}}+\frac{4}{x} \rho\left(p^{a}\right) p^{a / 2} \\
& -\frac{4}{\log x} \frac{\log p^{a}}{p^{a / 2+1}}-\frac{4}{\log x} \frac{\log p}{p^{a / 2+1}}+e\left(p^{a}\right),
\end{aligned}
$$

$$
\begin{aligned}
\sum_{q^{b} \leq x} c\left(p^{a}, q^{b}\right) x_{2}\left(q^{b}\right)= & \frac{6}{\log x} \frac{\log p^{a}}{p^{a / 2}}-\frac{3}{\log ^{2} x} \frac{\log ^{2} p^{a}}{p^{a / 2}}-\frac{3}{x} \rho\left(p^{a}\right) p^{a / 2} \\
& -\frac{6}{\log x} \frac{\log p^{a}}{p^{a / 2+1}}-\frac{6}{\log x} \frac{\log p}{p^{a / 2+1}}-\frac{6 \alpha}{\log ^{2} x} \frac{\log p^{a}}{p^{a / 2}}+e\left(p^{a}\right) .
\end{aligned}
$$

Using Lemmas 3 and 4, we obtain

$$
\begin{aligned}
\sum_{q^{b} \leq x} c\left(p^{a}, q^{b}\right) x_{3}\left(q^{b}\right)= & \frac{2(\alpha-\beta)}{\log x} \frac{1}{p^{a / 2}}-\frac{2(\alpha-\beta)}{\log x} \frac{1}{p^{a / 2+1}}-\frac{2}{x} \frac{1}{p^{a / 2}} \sum_{q^{b} \leq x / p^{a}} \rho\left(q^{b}\right) \\
& +\frac{2}{x} \frac{1}{p^{a / 2+1}} \sum_{q^{b} \leq x / p^{a}} \rho\left(q^{b}\right)+\frac{2}{x^{2}} p^{a / 2} \sum_{q^{b} \leq x / p^{a}} \rho\left(p^{a} q^{b}\right) \rho\left(q^{b}\right) q^{b} \\
& -\frac{2}{x^{2}} p^{a / 2} \sum_{q^{b} \leq x / p^{a}} \rho\left(p^{a+1} q^{b}\right) \rho\left(q^{b}\right) q^{b}
\end{aligned}
$$

We observe that

$$
\sum_{q^{b} \leq x} c\left(p^{a}, q^{b}\right) x_{4}\left(q^{b}\right)=\frac{4(\alpha-\beta)}{\log x} \frac{1}{p^{a / 2}} \sum_{x / p^{a}<q^{b} \leq x} \frac{1}{q^{b}}+e\left(p^{a}\right) .
$$

Finally we note that the contributions of $x_{i}, i=5,6,7$, are small, i.e., for such $i$,

$$
\sum_{q^{b} \leq x} c\left(p^{a}, q^{b}\right) x_{i}\left(q^{b}\right)=e\left(p^{a}\right)
$$


Therefore from (3.2)-(3.6) we see that the $p^{a}$ th component of $\mathbf{C x}-\mu \mathbf{x}$ is

$$
\begin{aligned}
& \sum_{q^{b} \leq x} c\left(p^{a}, q^{b}\right) x\left(q^{b}\right)-\left(-\frac{1}{2}+\frac{A}{\log x}\right) x\left(p^{a}\right) \\
&=\frac{6 \alpha-4 A}{\log ^{2} x} \frac{\log p^{a}}{p^{a / 2}}+\frac{6 A}{\log ^{3} x} \frac{\log ^{2} p^{a}}{p^{a / 2}}-\frac{4(\alpha-\beta)}{\log x} \frac{1}{p^{a / 2}} \sum_{x / p^{a}<q^{b} \leq x} \frac{1}{q^{b}} \\
& \quad-\frac{2}{x} \frac{1}{p^{a / 2}} \sum_{q^{b} \leq x / p^{a}} \rho\left(q^{b}\right)+\frac{2}{x} \frac{1}{p^{a / 2+1}} \sum_{q^{b} \leq x / p^{a}} \rho\left(q^{b}\right) \\
&+\frac{2}{x^{2}} p^{a / 2} \sum_{q^{b} \leq x / p^{a}} \rho\left(p^{a} q^{b}\right) \rho\left(q^{b}\right) q^{b} \\
&-\frac{2}{x^{2}} p^{a / 2} \sum_{q^{b} \leq x / p^{a}} \rho\left(p^{a+1} q^{b}\right) \rho\left(q^{b}\right) q^{b}+e\left(p^{a}\right) .
\end{aligned}
$$

We will estimate $\|\mathbf{C x}-\mu \mathbf{x}\|$. By Minkowski's inequality, $\|\mathbf{C x}-\mu \mathbf{x}\|$ is bounded by the norm of the vector whose typical component is given by the first seven terms in (3.7) plus the norm of the error vector e. As $\|\mathbf{e}\| \ll \log \log x / \log ^{3 / 2} x$, we will calculate the square of the norm of the main vector. The contribution of the first three terms in (3.7) is

$$
\begin{aligned}
& \frac{(6 \alpha-4 A)^{2}}{\log ^{4} x} \sum_{p^{a} \leq x} \frac{\log ^{2} p^{a}}{p^{a}}+\frac{12 A(6 \alpha-4 A)}{\log ^{5} x} \sum_{p^{a} \leq x} \frac{\log ^{3} p^{a}}{p^{a}}+\frac{36 A^{2}}{\log ^{4} x} \sum_{p^{a} \leq x} \frac{\log ^{2} p^{a}}{p^{a}} \\
& \quad-\frac{8(6 \alpha-4 A)(\alpha-\beta)}{\log ^{3} x} \sum_{p^{a} q^{b}>x} \frac{\log p^{a}}{p^{a}} \frac{1}{q^{b}}-\frac{48 A(\alpha-\beta)}{\log ^{4} x} \sum_{p^{a} q^{b}>x} \frac{\log ^{2} p^{a}}{p^{a}} \frac{1}{q^{b}} \\
& \quad+O_{A}\left(1 / \log ^{3} x\right) .
\end{aligned}
$$

Treating the first three sums by Lemma 1 and the next two sums by Lemma 2 , the above is

$$
\frac{A^{2}-4 A(\alpha-\beta)-30 \alpha^{2}+48 \alpha \beta}{\log ^{2} x}+O_{A}\left(1 / \log ^{3} x\right) .
$$

One can see that the contribution of the remaining terms in (3.7) is $L / \log ^{2} x+$ $O\left(\log \log ^{2} x / \log ^{3} x\right)$ for some constant $L$. Although we can calculate the value of $L$, we do not need it to prove the theorem. Hence

$$
\|\mathbf{C} \mathbf{x}-\mu \mathbf{x}\| \leq \frac{\sqrt{A^{2}-4 A(\alpha-\beta)+K}}{\log x}+O_{A}\left(\log \log ^{2} x / \log ^{2} x\right),
$$

where the new constant $K$ is $K=L-30 \alpha^{2}+48 \alpha \beta$, and so by (3.1) we conclude that there exists an eigenvalue $\nu$ such that

$$
\begin{aligned}
-\frac{1}{2} & +\frac{A-\sqrt{A^{2}-4 A(\alpha-\beta)+K}}{\log x}+O_{A}\left(\log \log ^{2} x / \log ^{2} x\right) \\
& \leq \nu \leq-\frac{1}{2}+\frac{A+\sqrt{A^{2}-4 A(\alpha-\beta)+K}}{\log x}+O_{A}\left(\log \log ^{2} x / \log ^{2} x\right) .
\end{aligned}
$$

From Kubilius's estimate for $\nu_{r}$ and the fact that $\nu_{r}$, for small $r$, are all distinct, we find that $\nu$ is equal to the smallest eigenvalue $\nu_{2}$ of the matrix $\mathbf{C}$ 
for all sufficiently large $x$. Seeing that $A-\sqrt{A^{2}-4 A(\alpha-\beta)+K}$ is positive when $A$ is large enough, we complete the proof of the upper bound inequality of the theorem. The final statement follows from

$$
\lim _{A \rightarrow \infty}\left(A-\sqrt{A^{2}-4 A(\alpha-\beta)+K}\right)=2(\alpha-\beta)=0.644 \ldots .
$$

To prove the other inequality in the theorem, first note that

$$
\frac{Q(\mathbf{x})}{\|\mathbf{x}\|^{2}}=\frac{\mathbf{x}^{t} \mathbf{C} \mathbf{x}}{\|\mathbf{x}\|^{2}}=\mu+\frac{\mathbf{x}^{t}(\mathbf{C} \mathbf{x}-\mu \mathbf{x})}{\|\mathbf{x}\|^{2}},
$$

and this is

$$
\leq-\frac{1}{2}+\frac{A+\sqrt{A^{2}-4 A(\alpha-\beta)+K}}{\log x}+O_{A}\left(\log \log ^{2} x / \log ^{2} x\right) .
$$

Secondly, we substitute the vector $\mathbf{x}$ in the expression of $P$ in $\S 1$ and a straightforwardly calculate to obtain

$$
\frac{P(\mathbf{x})}{\|\mathbf{x}\|^{2}}=1-\frac{e}{\log x}+O\left(\log \log x / \log ^{2} x\right)
$$

for some positive constant $e$. This ends the proof.

\section{ACKNOWLEDGMENT}

It is my pleasure to thank Professor Hugh Montgomery for the suggestion of this problem and a lot of precious conversations, and Professor Brian Conrey for his encouragement and comments during the preparation of this paper. I also thank the referee for helpful suggestions.

\section{REFERENCES}

1. M. de la Vallée-Poussin, Sur les valeur moyennes de certaines fonctions arithmetiques, Ann. Soc. Sci. Bruxelles 22 (1898), 84-90.

2. P. D. T. A. Elliott, Functional analysis and additive arithmetic functions, Bull. Amer. Math. Soc. 16 (1987), 179-223.

3. A. Hildebrand, An asymptotic formula for the variance of an additive function, Math. Z. 183 (1983), 145-170.

4. R. Horn and C. Johnson, Matrix analysis, Cambridge Univ. Press, Cambridge, 1985.

5. J. Kubilius, On the estimation of the second central moment for strongly additive functions, Liet. Mat. Rinkinys 23 (1983), 122-133. (Russian)

6. - On the estimation of the second central moment for any additive arithmetic functions, Liet. Mat. Rinkinys 23 (1983), 110-117. (Russian)

7. J. Lee, On the constant in the Turan-Kubilius inequality, Thesis, The University of Michigan, 1989.

8. C. M. Stein, On the Turán-Kubilius inequality, Technical Report No. 220, Stanford University, 1984.

Department of Mathematics, Oklahoma State University, Stillwater, Oklahoma 74078

Current address: Department of Mathematics, Kansas State University, Manhattan, Kansas 66506 